University of Wollongong

Research Online

Faculty of Engineering and Information

Faculty of Engineering and Information

Sciences - Papers: Part A

Sciences

$1-1-2010$

\title{
Ultrabroadband optical parametric chirped pulse amplifier system for single-cycle waveform synthesis
}

Shu-Wei Huang

Massachusetts Institute of Technology

Giovanni Cirmi

Massachusetts Institute of Technology

Jeffrey Moses

Massachusetts Institute of Technology

Kyung-Han Hong

Massachusetts Institute of Technology

Andrew Benedick

Massachusetts Institute Of Technolog

See next page for additional authors

Follow this and additional works at: https://ro.uow.edu.au/eispapers

Part of the Engineering Commons, and the Science and Technology Studies Commons

Research Online is the open access institutional repository for the University of Wollongong. For further information contact the UOW Library: research-pubs@uow.edu.au 


\title{
Ultrabroadband optical parametric chirped pulse amplifier system for single-cycle waveform synthesis
}

\author{
Abstract \\ We demonstrate synchronized few-cycle $800-\mathrm{nm}$ and $2-\mu \mathrm{m}$ pulse trains seeded from a single Ti:sapphire \\ oscillator, able to generate scalable, high-energy pulses lasting less than a single electric-field cycle. Such \\ pulses are attractive for high-field physics.

\section{Keywords} \\ single, system, amplifier, pulse, synthesis, chirped, waveform, parametric, optical, ultrabroadband, cycle \\ Disciplines \\ Engineering | Science and Technology Studies

\section{Publication Details} \\ Huang, S., Cirmi, G., Moses, J., Hong, K., Benedick, A., Chen, L., Li, E., Eggleton, B., Cerullo, G. \& Kartner, F. \\ X. (2010). Ultrabroadband optical parametric chirped pulse amplifier system for single-cycle waveform \\ synthesis. Lasers and Electro-Optics (CLEO) and Quantum Electronics and Laser Science Conference \\ (QELS), 2010 Conference on (pp. 1-2). United States: Institute of Electrical and Electronics Engineers. \\ Authors \\ Shu-Wei Huang, Giovanni Cirmi, Jeffrey Moses, Kyung-Han Hong, Andrew Benedick, Li-Jin Chen, Enbang \\ $\mathrm{Li}$, Benjamin J. Eggleton, Giulio Cerullo, and Franz X. Kartner
}




\title{
Ultrabroadband Optical Parametric Chirped Pulse Amplifier System for Single-Cycle Waveform Synthesis
}

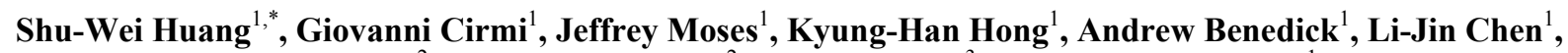 \\ Enbang Li $i^{2}$, Benjamin Eggleton ${ }^{2}$, Giulio Cerullo ${ }^{3}$ and Franz X. Kärtner ${ }^{1}$ \\ ${ }^{1}$ Research Laboratory of Electronics, Massachusetts Institute of Technology, 77 Massachusetts Ave, Cambridge MA-02139 USA \\ ${ }^{2}$ CUDOS ARC Centre of Excellence School of Physics, University of Sydney, Sydney, Australia \\ ${ }^{3}$ Dipartimento di Fisica, Politecnico di Milano, Piazza Leonardo da Vinci, 32 - 20133 Milano (MI), Italy \\ *Email: klkla@mit.edu
}

\begin{abstract}
We demonstrate synchronized few-cycle 800-nm and 2- $\mu \mathrm{m}$ pulse trains seeded from a single Ti:sapphire oscillator, able to generate scalable, high-energy pulses lasting less than a single electric-field cycle. Such pulses are attractive for high-field physics.

(c) Optical Society of America

OCIS codes: (190.4970) Parametric oscillators and amplifiers, (320.7110) Ultrafast nonlinear optics.
\end{abstract}

Advances in ultrafast optics over the last decade have led to the generation of close to single-cycle optical pulses with Carrier-Envelope Phase (CEP) control, i.e., one is able to generate a precisely defined electric field waveform [1]. These pulses are currently used to drive strong-field processes such as high-harmonic generation (HHG) and have recently led to efficient generation of isolated attosecond pulses as short as 80-as in the XUV range [2]. Nevertheless, the technique relies on a highly nonlinear process that is not scalable in energy.

Our work aims towards single-cycle optical pulse generation, via synthesis of few-cycle pulses from two ultrabroadband optical parametric chirped pulse amplifiers (OPCPAs) seeded by the same octave spanning Ti:Sapphire oscillator. Such pulses can be used to efficiently generate isolated attosecond pulses without the use of gating techniques [3]. Optical Parametric Amplifiers (OPAs) and OPCPAs have proved to be a workhorse for the generation of few-cycle optical pulses. Ultrabroadband OPCPA is the only demonstrated technique for producing few-cycle pulses with multi-terawatt peak power [4].

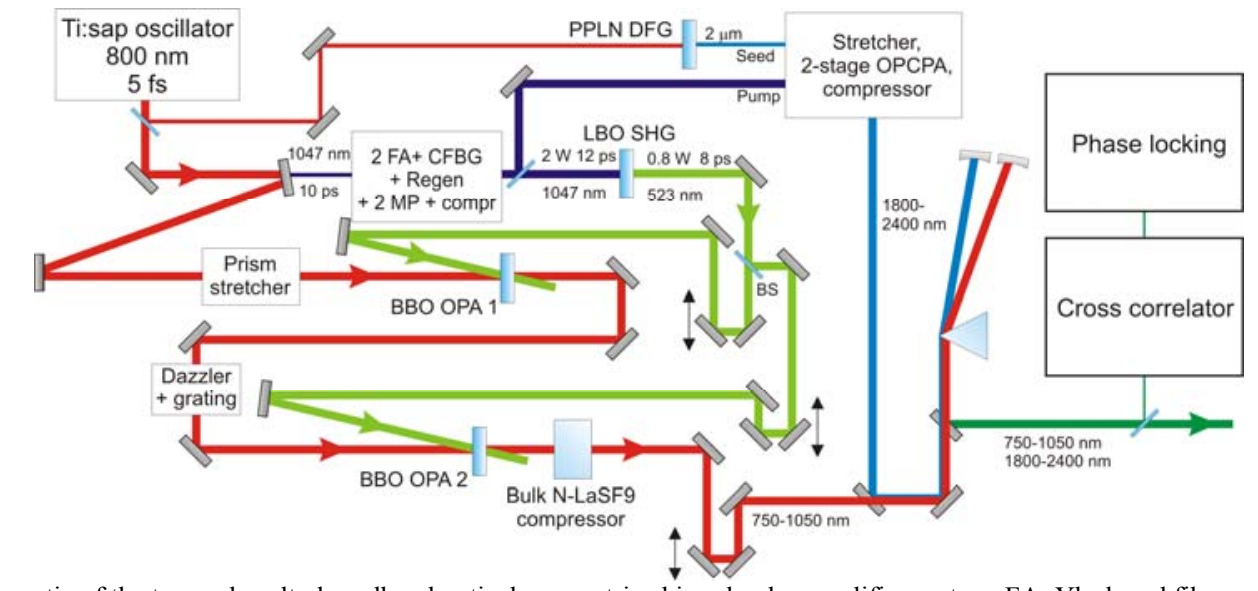

Fig. 1. Schematic of the two-color ultrabroadband optical parametric chirped pulse amplifier system. FA: Yb-doped fiber amplifier; MP: multi-pass slab amplifier; SHG: second harmonic generation.

The system starts with a Ti:Sapphire oscillator producing 5-fs pulses centered at $800-\mathrm{nm}$ with nJ-level energy per pulse and an $80-\mathrm{MHz}$ repetition rate. We have developed a Nd:YLF chirped pulse amplifier (CPA) as a pump source, which consists of a chirped fiber Bragg grating (CFBG) stretcher, a regenerative amplifier followed by two multi-pass slab amplifiers, and a diffraction grating compressor. A small fraction of the oscillator bandwidth around $1047 \mathrm{~nm}$ is used to seed the CPA. After the grating compressor, we obtain 3.5-mJ, 12-ps pulses for pumping the two OPCPAs. For the 800-nm OPCPA, a 2-mJ fraction of the 1047-nm pulse is frequency doubled in a Lithium Triborate (LBO) crystal and used to directly amplify the oscillator output. The seed is first stretched to 5 ps by a prism stretcher. Seed and pump interact in a double-pass configuration in a type-I, 5 -mm-long $\beta$-Barium Borate (BBO) crystal cut at $\theta=24^{\circ}$, with a $2.4^{\circ}$ pump-signal angle which guarantees a broad phase matching bandwidth in a non-collinear geometry. The gain of the first OPCPA stage is $\sim 10^{5}$, and the energy of the amplified pulses is $2 \mu \mathrm{J}$. The amplified pulse is then further stretched to $6.2 \mathrm{ps}$ by an acousto-optic programmable dispersive filter (AOPDF: 


\section{CWA4.pdf}

DAZZLER, Fastlite) and a grating stretcher, allowing for simultaneous optimization of energy conversion, amplification bandwidth, and signal-to-noise ratio in the power amplifier stage. The pulse is then amplified to $25 \mu \mathrm{J}$, in the same kind of crystal as the first stage, and compressed in a bulk N-LaSF9 block. The transform-limited pulse duration is $6.5 \mathrm{fs}$, and the final spectrum is shown in Fig. 2(a).

The 2- $\mu \mathrm{m}$ OPCPA follows the design method in [5]. Passively CEP-stabilized broadband seed pulses are generated by intra-pulse difference frequency generation (DFG), stretched by a bulk silicon block to 5 ps and preamplified to $1.5 \mu \mathrm{J}$ in the first OPCPA stage. The pre-amplified pulse is further stretched to $9.5 \mathrm{ps}$ by an infrared AOPDF, amplified to $30 \mu \mathrm{J}$, and then compressed in a broadband anti-reflection coated quartz glass block (Suprasil 300). $1 \mathrm{~mJ}$ of the available $1047-\mathrm{nm}$ pump energy is used between the two stages. Fig. 2(b) shows the final spectrum, corresponding to a pulse width of $23 \mathrm{fs}$.

The first step of the single-cycle pulse synthesis is to recombine the two beams. To do this we send the two pulses into a prism and focus them back onto the prism at the same spatial point. As described earlier, since the OPCPAs and pump laser system are all seeded by a single octave-spanning Ti:Sapphire oscillator, the OPCPA pulses are already synchronized. However, there may be slow drifts in the synchronization due to environmental fluctuations. The timing between the two pulses will be detected by a balanced cross-correlator [6], and feedback control will be implemented to achieve tens of attosecond synchronization. The inclusion of an AOPDF in each of the two OPCPAs allows independent control of the dispersion of each pulse, allowing full control and optimization of the synthesized waveform.

To complete the synthesis, the CEP of each amplifier must be stable and the relative CEP between the two pulses also has to be locked. We have demonstrated that the 2- $\mu \mathrm{m}$ OPCPA is passively phase stabilized [5] and the 800-nm OPCPA can be actively stabilized. The relative CEP will be locked by heterodyne beating of the two pulses. Both timing and phase locking will be performed in an interferometrically stable arrangement with respect to the synthesized pulse to avoid possible drifts.

Fig. 2(c) shows the calculated electric field profile of the synthesized pulse. The electric field waveform has a distinct central lobe that is sub-cycle in duration, with only 0.85 -cycles of the center frequency within the FWHM of the electric-field amplitude profile. The FWHM of the intensity profile is only 0.55 cycles in duration. The synthesized pulses will allow for nonlinear light-matter interactions dominated by one half-cycle of the electric field waveform, such as the study of ionization processes, efficient isolated attosecond pulse generation by HHG, and direct laser acceleration of electron bunches. For some of the latter processes it is preferred to scale the pulse energy to the multi-mJ level, which we intend to do with a higher energy and power pump laser system based on cryogenically-cooled Yb:YAG [7].
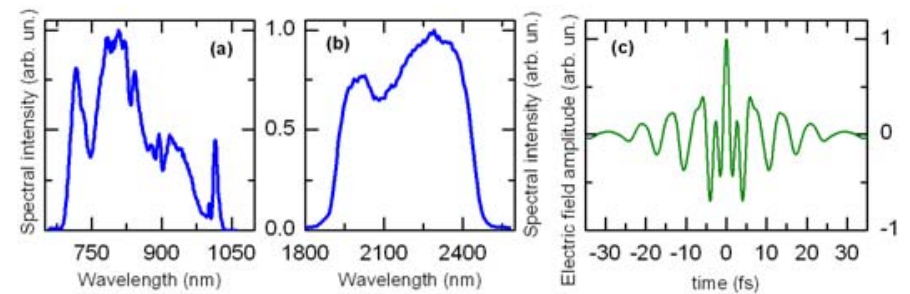

Fig. 2. (a) Amplified signal spectrum of the 800-nm OPCPA. (b) Amplified signal spectrum of the 2- $\mu$ m OPCPA. (c) Electric field waveform of a synthesized pulse.

In summary, the overall system discussed in this paper shows a path towards an energy-scalable single-opticalcycle waveform synthesizer.

[1] A. L. Cavalieri, E. Goulielmakis, B. Horvarth, W. Helml, M. Schultze, M. Fiess, V. Pervak, L. Veisz, V. Yakovlev, M. Uiberacker, A. Apolonski, F. Krausz, and R. Kienberger, "Intense 1.5-cycle near infrared laser waveforms and their use for the generation of ultra-broadband soft-x-ray harmonic continua," New J. Phys., 9, 242 (2007).

[2] E. Goulielmakis, M. Schultze, M. Hofstetter, V. S. Yakovlev, J. Gagnon, M. Uiberacker, A. L. Aquila, E. M. Gullikson, D. T. Attwood, R. Kienberger, F. Krausz, and U. Kleineberg, "Single-Cycle Nonlinear Optics," Science, 320, 1614 (2008).

[3] H. Mashiko, S. Gilbertson, C. Q. Li, S. D. Khan, M. M. Shakya, E. Moon, and Z. H. Chang, "Double optical gating of high-order harmonic generation with carrier-envelope phase stabilized lasers," Phys. Rev. Lett., 100, 103906 (2008).

[4] F. Tavella, A. Marcinkevičius, and F. Krausz, "90 mJ parametric chirped pulse amplification of 10 fs pulses", Opt. Expr., 14, 12822 (2006). [5] J. Moses, S.-W. Huang, K.-H. Hong, O. D. Mücke, E. L. Falcão-Filho, A. Benedick, F. Ö. Ilday, A. Dergachev, J. A. Bolger, B. J. Eggleton, and F. X. Kärtner, "Highly stable ultrabroadband mid-IR optical parametric chirped-pulse amplifier optimized for superfluorescence suppression", Opt. Lett., 34, 1639 (2009).

[6] T. R. Schibli, J. Kim, O. Kuzucu, J. T. Gopinath, S. N. Tandon, G. S. Petrich, L. A. Kolodziejski, J. G. Fujimoto, E. P. Ippen, and F. X. Kaertner, "Attosecond active synchronization of passively mode-locked lasers by balanced cross correlation," Opt. Lett., 28, 947 (2003).

[7] K.-H. Hong, J. Gopinath, D. Rand, A. Siddiqui, S.-W. Huang, E. Li, B. Eggleton, T. Y. Fan, and F. X. Kärtner, "High-energy, picosecond, cryogenic Yb:YAG chirped-pulse amplifier at kHz repetition rates for OPCPA pumping," submitted to CLEO 2010. 